\title{
Coverillustration \\ We hardly know those eyes
}

$\mathrm{T}$ he electromagnetic spectrum of the sun produces a peak of energy in the region of the shorter wavelengths of the visible spectrum. It is no surprise, then, that life on earth has capitalised on this energy source for growth and sensory adaptations. But there are other portions of the spectrum that provide useful sensory information for those organisms capable of its recognition.

Rowley's palm viper (Bothriechis rowleyi) seen on this month's cover, is an arboreal, montane representative of Crotalinae, an elite subfamily of snakes capable of radiant thermal detection and poisonous prey dispatch. A more primitive but related family, Boidae, that includes the boas, also possesses infrared detection abilities, but these species have no poison.

The nerve endings in the infrared sensitive pits located anterior to the eyes in these pit vipers are capable of detecting temperature differences as subtle as $0.05^{\circ} \mathrm{C}$ at the surface of the receptor. These magnificent snakes can even strike, based on thermal clues alone, as the pits face forward with overlapping fields, thus making the thermal sensitivity "stereoscopic" in nature. These ultrasensitive bolometers are truly stunning, because they combine thermal isolation and extreme vascularisation to create an extraordinarily sensitive organ, capable of sensing a $10^{\circ} \mathrm{C}$ difference as far away as $20-25 \mathrm{~cm}$. These pit organs are bimodal receptors absorbing energy in two regions of the infrared spectrum, specifically in the $3-5 \mu \mathrm{m}$ and $8-12 \mu \mathrm{m}$ range. This sensitivity matches the radiant spectrum of their principal preyrodents. The infrared sensitive neurons travel to the trigeminal ganglion, and then to the lateral descending trigeminal tract in the medulla, unique to snakes. The neurons then travel to and merge with the visual input in the optic tectum. The snakes are then able to "visualise" their environment with at least two different portions of the electromagnetic spectrum mediated through an entirely different "visual" pathway. Hence, the pit vipers do not rely upon a single sense to apprehend their prey. In addition to using the infrared and visible spectrum, the pit vipers integrate the chemosensory input from the vomeronasal organ, the organ of Jacobson to fully locate their prey. Their flicking tongues provide the molecular input to that peculiar organ on the upper palate, and do so with directional clues since the tongue is forked. Even during the strike with its mouth open, the snake can follow its victim since it has thermal receptors scattered in the oropharynx. Thermal visualisation relies upon the contrast at the edge of the patterns and is analogous to human vision. Similar to human vision, the

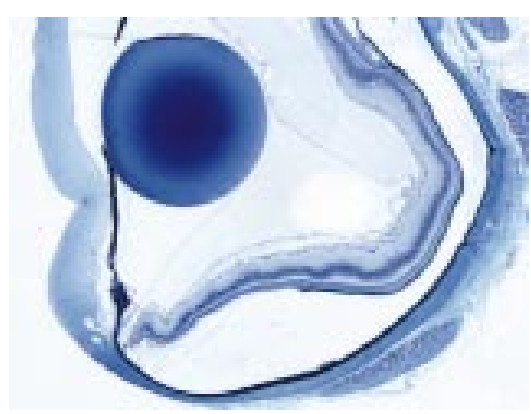

Figure 1 Histopathology slide of eye of sharp-tailed snake. Note round crystalline lens, shaped much like a piscine lens. (Histopathology by Thomas N Blankenship, PhD.)

perceived thermal display exhibits a phenomenon known as Mach bands. Mach bands represent a special focus of brightness contrast perceived by our brain but not really present at the edge of a brighter visual stimulus. Our visual system processes the edge as brighter because of these successful Mach bands. The pit vipers apparently have the same perception of Mach bands at the edge of thermally different objects.

Pit vipers do not depend upon vision for predator capture to the same degree as some other species such as birds, and prey capture can be performed in total darkness. Nevertheless, the visual and sensory mechanisms of these beautiful and dangerous animals are interesting, are used to increase accuracy, and may be the principal sensory mechanism on diurnal hunts.

These three senses then provide a unique view of prey and extend the potential for predation from daytime to the total darkness of a subterranean burrow.

The eyes of snakes are interesting but are not visually exceptional. Accommodation is accomplished by an anterior movement of the lens. Snake eyes adjust for distance when the lens is at rest, but with accommodation the crystalline lens moves forward and can focus at a nearer point, accommodating for near, like us. The pit vipers are not among those few snake species that have a fovea or even a macula, but as mentioned above they have other robust sensory mechanisms.

The vertical pupil, seen in other nocturnal predators, can constrict to an almost invisible slit in pit vipers to protect a rod-rich retina, especially during the day. The retina of Bothriechis rowleyi is unknown. The iris musculature is striated and fast, much like birds, so that the snake can create a very narrow slit to limit light and feed diurnally, if necessary.

Extinction is a natural evolutionary process to which all species, including our own, will succumb. Unfortunately, in geological history, humans will probably be the cause of a major extinction the likes of which the earth has not seen since the Permian extinction. This neotropical pit viper, Bothriechris rowleyi (Rowley's palm viper), is native to the cloud forests of Oaxaca and Chiapas in central Mexico and was only recently discovered. But, with habitat destruction other individuals of this striking animal have not been found and it may be extinct in the wild. This arboreal, mostly nocturnal, snake is threatened with extinction, and we barely know its name.

I R Schwab

University of California Davis, Department of Ophthalmology, 4860 " $Y$ " Street, Suite 2400 , Sacramento CA. 95817, USA; irschwab@ucdavis.edu

Photograph taken at the San Antonio Zoo, by the author. 